IMPACT OF A MULTIPARAMETER POINT-OFCARE TEST (POCT) ANALYZER ON BILIRUBIN - ELECTROLYTE MONITORING AND BLOOD TRANSFUSIONS IN A NICU

\author{
L. Mahieu ${ }^{1}$, H. Cluckers², P. Rombouts ${ }^{2}$, \\ V. Van Hoof ${ }^{2}$ \\ ${ }^{1}$ Pediatrics Div. Neonatology, ${ }^{2}$ Clinical Chemistry, \\ Univ. Hosp. Antwerp, Edegem, Belgium
}

Introduction: Since 2008, we introduced a multiparameter Point of Care testing (POCT) analyzer (Cobas-b221, Omni-S, Roche, Belgium). In comparison to central laboratory performed blood tests, POCT requires less blood (100-150 microL vs. 500 microL).

Aim: To assess the impact of POCT on total number of tests performed and blood transfusions performed in our NICU.

Methods: The number of bilirubin/ blood electrolyte tests performed by POCT and central laboratory performed tests were retrieved from the hospital information system. The number of tests and the number of transfusion were compared for the 2 periods, before (2006-2007) and after (2008-2009) introduction of POCT in the NICU.

Results: While the laboratory performed electrolyte tests decreased from 6863 to $4673(-32 \%)$ from period-1 to period-2, the total electrolyte tests performed increased from 7067 to 7771 (+10\%). While the central laboratory tests for bilirubin decreased from 5177 to $3743(-27,7 \%)$, the total bilirubin tests performed increased from 5196 to 6196 tests $(+20 \%)$.

The estimated blood volume loss decreased for bilrubin from $3055 \mathrm{ml}$ to $2121 \mathrm{ml}(-31 \%)$ and for electrolytes from $3455 \mathrm{~mL}$ to $2801 \mathrm{~mL}(-19 \%)$.

The number of transfusion per infant decreased from $2.53+/-4.0$ to $1.57+/-2.8$ (change $-48 \%$, p $=0.014$ ) in infants $<1500 \mathrm{~g}$, from $0.19+/-0.75$ to $0.16+/-0.65$ (change $-16 \%, p=0.88$ ) in those > $1500 \mathrm{~g}$.

Conclusion: Despite increased monitoring for bilirubin and electrolytes after introduction of POCT, there was a significant reduction in blood loss and blood transfusions in the NICU.

\section{“MATERNALIZING” THE NICU: EFFECTS OF MATERNAL SOUNDS ON GROWTH AND DEVELOPMENT IN PREMATURE INFANTS}

\author{
A. Lahav ${ }^{1,2}$, E. Wachman ${ }^{1}$, R. Insoft ${ }^{1}$, S. Ringer ${ }^{1}$ \\ ${ }^{1}$ Newborn Medicine, Brigham and Women's \\ Hospital, Harvard Medical School, '2Pediatrics, \\ Mass General Hospital for Children, Boston, MA, \\ USA
}

Premature infants are at high risk for developing lifelong disabilities. There is a concern that the sound environment in the NICU increases the vulnerability of the infant and increases developmental complications. NICU noise typically exceeds recommended levels and masks other meaningful sound from reaching the infant. Here we present a pilot study looking at the effects of Soothing Maternal Sounds (SMS) vs. Routine NICU Sounds (RNS) on short-term outcomes. We performed a randomized controlled trial in premature infants born between 2532 weeks' gestation. The SMS group was provided with a recording of their own mother's voice and heartbeat throughout their NICU hospitalization. Maternal sounds were transmitted to the infant's incubator via a specially-designed innovative micro audio technology. Infants in the RNS group received standard NICU care. Data collected daily from the infant's bedside monitor included heart rate, respiratory rate, oxygen saturation level, blood pressure, and presence of apnea/bradycardia episodes. Our results provide preliminary evidence that early exposure to maternal sounds may increase physiological stability in the neonatal period. Additional growth outcomes including weight gain, height, and head circumference are discussed. This data will help us determine whether modulating the sound environment in the NICU can increase growth and short-term physiological stability in premature infants. Future developments derived from this study will enable us to improve NICU care and reduce complications of prematurity. 